\title{
Intertwined Digital Rays in Discrete Radon Projections Pooled over Adjacent Prime Sized Arrays
}

\author{
Imants Svalbe and Andrew Kingston \\ Center for X-ray Physics and Imaging \\ School of Physics and Materials Engineering \\ Monash University, VIC 3800, AUS
}

\begin{abstract}
Digital projections are image intensity sums taken along directed rays that sample whole pixel values at periodic locations along the ray. For $2 \mathrm{D}$ square arrays with sides of prime length, the Discrete Radon Transform (DRT) is very efficient at reconstructing digital images from their digital projections. The periodic gaps in digital rays complicate the use of the DRT for efficient reconstruction of tomographic images from real projection data, where there are no gaps along the projection direction. A new approach to bridge this gap problem is to pool DRT digital projections obtained over a variety of prime sized arrays. The digital gaps are then partially filled by a staggered overlap of discrete sample positions to better approximate a continuous projection ray. This paper identifies primes that have similar and distinct DRT pixel sampling patterns for the rays in digital projections. The projections are effectively pooled by combining several images, each reconstructed at a fixed scale, but using projections that are interpolated over different prime sized arrays. The basis for the pooled image reconstruction approach is outlined and we demonstrate the principle of this mechanism works.
\end{abstract}

Keywords: Discrete Radon transform, tomographic image reconstruction.

\section{Introduction}

The Discrete Radon Transform (DRT) maps discrete image data $I(x, y)$ into discrete digital projections $R(t, m)$ that closely resemble continuous space integral Radon transforms [1]. The inherently discrete nature of the sampling and representation of projections makes the DRT an attractive tool to transform and interpret digital data [2]. In contrast with the case for continuous space, the digital projection mechanism requires no data interpolation, as each digital projection (labelled by index $m$ ) sums whole pixel values sampled along its ray direction. The samples are oriented at integer array displacements of $x_{m}$ horizontally and $y_{m}$ vertically on the lattice at each translate position $(t)$. Arrays of prime size 3] generate unique pixel sampling patterns for each DRT projection. This means that digital images can be projected and reconstructed exactly with 
the DRT using only simple (and hence fast) addition operations. Basic properties of digital projections for the DRT are reviewed in [45].

The prime based DRT has been applied successfully for the reconstruction of tomographic images from projections based on x-ray transmission [6]. In [7] linear 1D interpolation was used to match each continuous integral ray to a corresponding discretely sampled digital ray in a DRT with expanded translates, called $k$-space, $R(k, \theta)$. The inverse DRT was then used to reconstruct the image using the digital projections inferred from the analogue projection data, after mapping $R(k, \theta)$ back to $R(t, m)$. The reconstructed image size is a free variable in such a process. The major disadvantage of this DRT based inversion of analog projections is that accurate image reconstruction requires a very large final image size (or, at best, calculation of a sub-sampled result based a large image). The size of the gaps between samples on digital rays scales as the square root of the image size [4], so that, in increasingly large images, the finite gaps have diminishing importance. The computational efficiency of the DRT method is, however, rapidly overwhelmed by the additional computation required to reconstruct large format digital images.

In this paper, we look at reconstructing images using the same DRT method, but by applying it over a relatively small range of neighbouring prime array sizes, to avoid the need to reconstruct large arrays.

Linear interpolation is a poor approximation to match digital and continuous rays. Direct interpolation from the rays of a sinogram into $k$-space at each projection angle is complicated by the jumbled ordering and variable spatial overlap of the digital rays. A more direct approach to solving this interpolation problem is developed in 8]. In this paper, the aim is to compensate for the poor approximation of linear interpolation by pooling the reconstructed images derived from appropriately resampled digital projections.

Section 2 establishes the link between the prime array size and the pattern of digital ray sampling for any given digital projection. Section 3 shows that the pattern of sampling for a projection $x_{m}: y_{m}$ repeats for primes $p^{\prime}=p \pm x_{m} y_{m}$ and that this repetition limits the range of image array sizes that can be usefully pooled. Section 4 shows examples of digital projections based on different sized images. Comparative image reconstruction results using the pooled projection approach are given in section 5. followed by discussion of the limitations of this technique and conclusions in section 6 .

\section{Wrap Factors on Prime Arrays}

A digital projection with label $m$ samples image pixels on adjacent rows of the image data that are always $m$ horizontal units apart, so that the pixels located at $(x, y)$ and $(x+m, y+1)$ are always part of the same digital projection. We take $(0,0)$ to be the image origin, with positive displacements in $x$ increasing to the left with $y$ increasing in the downwards direction. Wherever $(x+m) \geq p$, the displacement is wrapped modulus $p$ to a new displacement on the same row. Each digital projection $m$ is comprised of a set of parallel segments or digital rays. These rays link the nearest neighbour pixels of a projection. The samples in 
a ray for projection $m$ are located at regular intervals $x_{m}$ units apart horizontally and $y_{m}$ units apart vertically on a square image array of size p. The sample gap, given by $d_{m}^{2}=x_{m}^{2}+y_{m}^{2}$, is the minimum distance between samples along that ray direction. The $x_{m}$ and $y_{m}$ values must be relatively prime, 9] outlines how the set of $x_{m}$ and $y_{m}$ values for any $p$ are drawn from the Farey series in number theory. The perpendicular separation between digital rays is given by $p / d_{m}$ with the horizontal offset between wrapped rays being $p / y_{m}$.

We are interested in $0<m<p$, as $m=0$ and $m=p$ are defined respectively as row and column projections. The integer variable $t, 0 \leq t<p$, defines the horizontal translation of a digital projection. For square lattice arrays, with $x_{m}<y_{m}$, there are four symmetric digital projections, $m_{0}, m_{1}, m_{2}$ and $m_{3}$, corresponding to $x_{m}: y_{m}, y_{m}: x_{m},-x_{m}: y_{m}$ and $-y_{m}: x_{m}$ with projection angles $\theta_{m}, 90-\theta_{m}, 90+\theta_{m}$ and $180-\theta_{m}$. It can be shown that $m_{3}=p-m_{0}$ and $m_{2}=p-m_{1}$ as these projections form complementary angles. The symmetric projections for each $x_{m}: y_{m}$ are important as they share symmetric patterns of pixel sampling. The values $x_{m}$ and $y_{m}$ are solutions of the digital linear projection equations [4], hence

$$
\begin{aligned}
& m_{0} y_{m}=\alpha_{0} p+x_{m} \\
& m_{1} x_{m}=\alpha_{1} p+y_{m} \\
& m_{2} x_{m}=\alpha_{2} p-y_{m} \\
& m_{3} y_{m}=\alpha_{3} p-x_{m},
\end{aligned}
$$

where the four constants $\alpha_{i}$ are positive integers indicating how many times the translation wraps around the array of size $p$ to get to the nearest ray sample location. For example, (1a) means that to project from the sample at $(0,0)$ to the nearest digital ray sample at $\left(x_{m}, y_{m}\right)$ in $y_{m}$ horizontal steps of $m_{0}$, requires $\alpha_{0}$ wraps around the right edge of the image of size $p$.

Figure 1 shows an example where $x_{m}=2, y_{m}=13$ for $m=422$ on a 457 by 457 array. The rays wrap 12 times for the samples to be nearest neighbours.

The points sampled by the digital projection $x_{m}: y_{m}$ are characterised completely by some $m_{0}$ for a particular $p$, that is $\left(x_{m}, y_{m}\right) \Leftrightarrow\left(m_{0}, p\right)$. Whilst there are many possible ways to have $\alpha_{0}$ wraps on an array of size $p$, the set $\left\{\alpha_{i}\right\}$ described by (1a-1d) defines a particular and distinct pattern of sampling of the digital rays across the image space to form that projection. Combining (1a $-1 \mathrm{~d}$

$$
\begin{aligned}
& \alpha_{0}+\alpha_{3}=y_{m} \\
& \alpha_{1}+\alpha_{2}=x_{m},
\end{aligned}
$$

we see that each $\left\{\alpha_{i}\right\}$ characterises an $x_{m}$ and $y_{m}$ pixel sample pattern independently of $p$. For the $2: 13$ ray, $\left\{\alpha_{i}\right\}=\{12,1,1,1\}$.

The values taken by the $\left\{\alpha_{i}\right\}$ in $2 \mathrm{a}-2 \mathrm{~b}$ are further restricted, as $\alpha_{1}$ and $\alpha_{2}$ cannot be factors of $x_{m}$ and, similarly, $\alpha_{0}$ and $\alpha_{3}$ cannot be factors of $y_{m}$. To prove this, assume, for example, that $x_{m}=j \alpha_{1}$. Then equation (1b) implies that $\alpha_{1}$ is also factor of $y_{m}$. For the gap $d_{m}$ to be a minimum distance, however, $x_{m}$ 


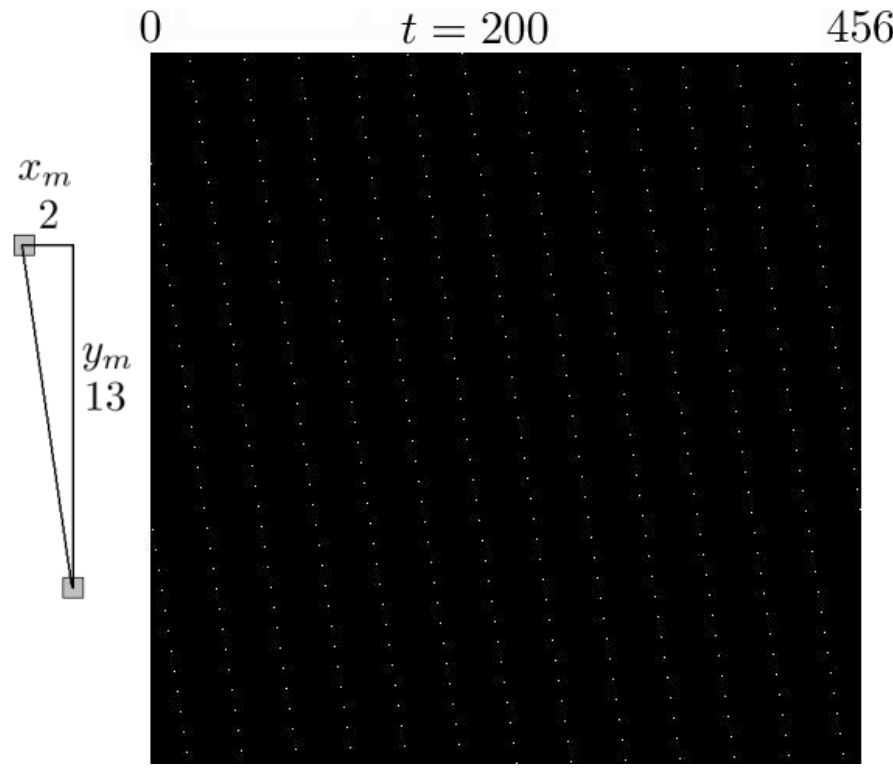

Fig. 1. Pixel locations (white dots) in a 457 by 457 image array that are sampled by the digital projection 2:13 $\left(m_{0}=422\right)$, for translate $t=200$. Digital rays follow the lines joining nearest neighbour pixels with $\alpha_{0}=12$.

and $y_{m}$ must be relatively prime, so the assumption must be false. For similar reasons, equation (2b) implies $\alpha_{1}$ and $\alpha_{2}$ must be relatively prime, as must be $\alpha_{0}$ and $\alpha_{3}$. Then, like the ordered set of $x_{m} / y_{m}$ fractions, $\left\{\alpha_{1} / \alpha_{0}, x_{m} / y_{m}, \alpha_{2} / \alpha_{3}\right\}$ also form a Farey-like sequence of relatively prime ratios [9], with $x_{m}$ and $y_{m}$ being mediants of the wrap factors.

\section{Primes with Common Wrap Factors}

Each allowed $\left\{\alpha_{i}\right\}$ defines a distinct pattern of sampling for the digital rays that comprise the projection $x_{m}: y_{m}$ of an image with size p. An array of size $p^{\prime}$ that has the same $\left\{\alpha_{i}\right\}$ will have the same relative pixel sampling pattern for the projection $x_{m}: y_{m}$, but with the separation of digital rays scaled by $p^{\prime} / p$.

Let the image array size change from $p$ to $p^{\prime}$, with $p$ and $p^{\prime}$ having the same $\left\{\alpha_{i}\right\}$ for projection $x_{m}: y_{m}$. Then equation (1a) becomes $m_{0}^{\prime} y_{m}=\alpha_{0} p^{\prime}+x_{m}$. Defining $\Delta m_{0}=m_{0}^{\prime}-m_{0}$ and $\Delta p=p^{\prime}-p$, then $\Delta m_{0}$ and $\Delta p$ are related by

$$
\Delta m_{0} y_{m}=\alpha_{0} \Delta p,
$$

and similarly,

$$
\Delta m_{1} x_{m}=\alpha_{1} \Delta p
$$

Equation (3a) requires that $\Delta p=n y_{m}$ for some integer $n=\Delta m_{0} / \alpha_{0}$, as $\Delta m_{0}$ is always an integer and $\alpha_{0}$ is not a factor of $y$. Equation (3b) requires 


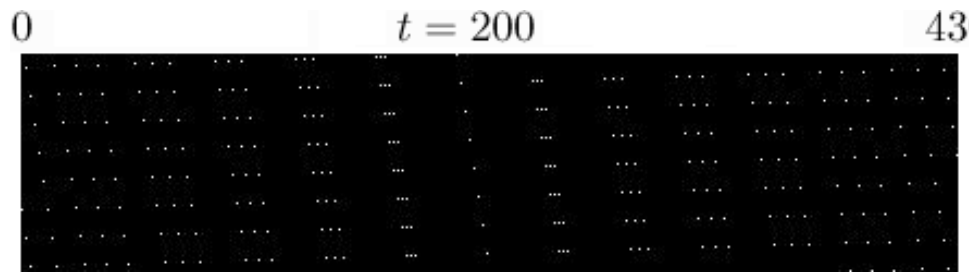

Fig. 2. Pooled pixel locations (white dots) for three array sizes, $457-26\left(m_{0}=398\right)$, $457\left(m_{0}=422\right)$ and $457+26\left(m_{0}=150\right)$, sampled by the digital projection $2: 13$, each with $t=200$. The array shown is a 431 by 100 image subset, with common origin $(0,0)$. The digital rays for the three array sizes have the same sampling pattern scaled to the width of each array.

that $\Delta p=n^{\prime} x_{m}$ for some integer $n^{\prime}$. Taken together, these constraints mean that the lowest possible value of $\Delta p$ for $p$ and $p^{\prime}$ to have the same $\left\{\alpha_{i}\right\}$ is then $\Delta p=x_{m} y_{m}$. Hence

$$
p^{\prime}=p \pm x_{m} y_{m}
$$

defines the array size nearest to $p$ that has the same $\left\{\alpha_{i}\right\}$ for the digital projection $x_{m}: y_{m}$. If $x_{m}$ and $y_{m}$ are odd, then, since $p$ is odd, the nearest prime $p^{\prime}$ with the same wrap factors is at least $p^{\prime}=p \pm 2 x_{m} y_{m}$. The value of $p^{\prime}$ given by (4) may not be a prime number.

Figure 2] shows that the 2:13 digital rays for the three arrays sizes, 457, 457士 26 , each with the same $\left\{\alpha_{i}\right\}$, have the same pixel sampling pattern, in proportion to the array width.

\section{Coincident Projection Segments}

Suppose the translate of one ray of a digital projection at $x_{m}: y_{m}$ in an image of size $p$ is aligned with the translate of one ray at $x_{m}: y_{m}$ in the size $p^{\prime}$ image. If $p$ and $p^{\prime}$ have the same $\left\{\alpha_{i}\right\}$, then all of the samples along the aligned rays match exactly within the array of size $p$, as shown in Figure2.

The perpendicular separation between digital rays is $p / d_{m}$ and the horizontal separation of the rays is $p / y_{m}$. The digital rays immediately adjacent to the aligned ray for array sizes of $p$ and $p^{\prime}=p+x_{m} y_{m}$ will have a perpendicular difference in separation, $d$, given by $d=\left(p^{\prime}-p\right) / d_{m}$, so that

$$
d=\frac{x_{m} y_{m}}{\sqrt{x_{m}^{2}+y_{m}^{2}}} .
$$

The integer horizontal displacement of the rays beside the coincident ray is $\pm x_{m}$. These displaced rays form part of a new projection translated by $x_{m}$ away from the ray with the gaps we are trying to fill, so that blending arrays with $p^{\prime}=p \pm x_{m} y_{m}$ will not fill the gaps. However all of the primes between $p$ and $p^{\prime}$ will have a perpendicular offset less than that given by (5) and will have pixels that sample the space between the original ray samples and those of the bounding 


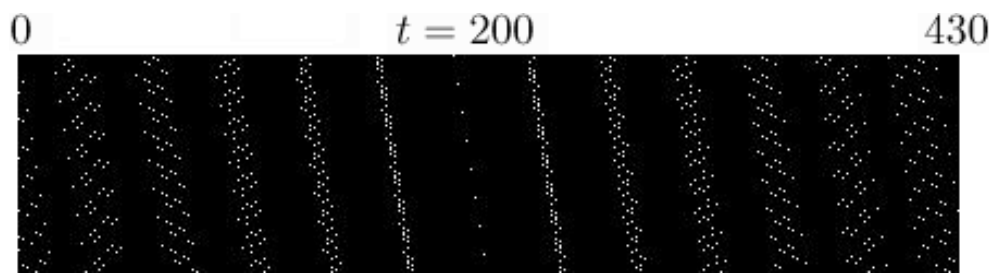

Fig. 3. Pooled digital rays for the projection $2: 13$, aligned at $t=200$, for the nine prime image array sizes $431<p<483$, shown as a 431 by 100 array subset, with $(0,0)$ as common origin.

rays for $p \pm x_{m} y_{m}$. As the array sizes are chosen to be prime, the sample locations for each blended digital ray will be unique. For those parallel digital rays further away from the aligned ray, the separation between rays belonging to $p$ and those belonging to $p^{\prime}$ become progressively more and more out of alignment (the $n^{\text {th }}$ parallel ray from the aligned set has a horizontal spread of $\pm n x_{m}$ ).

Figure 3 shows, as an example, the pattern of pixels sampled by the nine prime array sizes between 431 through 483, for the projection 2:13, each drawn with a common translate, $t=200$. The image shown is a 431 by 100 subset of the nine image arrays, with $(0,0)$ as a common origin. Here $x_{m} y_{m}=26$, so the array sizes are chosen to lie inside the range $457 \pm 26$. The samples for the ray through $t=200$ match exactly for all these primes. The size of the gap between sample points along the ray direction is $d_{m}=\sqrt{173} \approx 13.15$. The adjacent rays immediately either side of the aligned ray at $t=200$ have 9 sample points inside $\pm d$, where here $d \approx 1.98$ pixel units (the horizontal separation is $x_{m}=2$ pixel units). The nine sample locations are distinct and randomly spread inside the area of size $2 d d_{m}$. In this example, the nine pooled projections fill about $1 / 5$ of the area bounded by the gap distance between digital ray pixel samples and the limiting rays set by $p \pm x_{m} y_{m}$.

As $p^{\prime}=p \pm x_{m} y_{m}$ sets limits for those primes with digital rays that would at least partially fill the gap between digital samples for $p$, it matters how many primes fall between $p-x_{m} y_{m}$ and $p+x_{m} y_{m}$. Each of those primes is guaranteed to have a different $\left\{\alpha_{i}\right\}$ to that for $p$. When more primes lie within this range, the gap is filled by more pixels. This results in a better approximation by the pooled digital rays to an integral projection passing through the same image space. As the number of primes lying within $\Delta p$ will vary with $p$, not all possible $\left\{\alpha_{i}\right\}$ values will necessarily occur. For the projection $2: 13$, only 3 of the 12 possible distinct $\left\{\alpha_{i}\right\}$ sets do not occur between 431 and 483. Some reconstructed image arrays will pad out gaps in given projections better than others, simply because more primes fall inside the same $\Delta p$ interval.

\section{$5 \quad$ Reconstruction of Images}

The pooling of digital rays as shown in Figure 3 enables the sum of these rays to be more correctly identified with the continuous space rays in real projection 
data at the corresponding angle and translation. Figure 1 makes it clear why linear interpolation works so poorly with the DRT method when reconstructing a single image using a small array size.

Projections can be pooled in image space rather than in $k$-space or $t$-space. If images are reconstructed at a constant scale, but from data sourced over arrays of different prime size, then the same process of staggered ray sampling occurs, but we see the effects in the reconstructed image rather than in the interpolated projections.

If the original real data sinogram is first padded with zeros and then reconstructed using the method described in [7], the result is an image at the same physical scale as the unpadded data, but in a larger frame. Adding several reconstructed images that are appropriately padded, cropped and registered, emulates the pooling of digital rays in reducing the effects of the ray gaps. The method to achieve the appropriate image scaling is described next.

A sinogram comprised of $N$ rays at $M$ angles is first reconstructed to an image of size $p$ by $p$. The same sinogram, padded symmetrically left and right by $N p^{\prime} /(2 p)$ zeros (where $p^{\prime}>p$ ), can be used to reconstruct an image of size $p^{\prime}$ by $p^{\prime}$ but will retain the image data at the same physical scale as for the $p$ by $p$ image. Averaging these two images after registration (shifting the origin by $\left(p^{\prime}-p\right) / 2$ in $x$ and $\left.y\right)$ will be equivalent to blending the digital rays as sampled over $p$ and $p^{\prime}$. To fill as much of the gap in the digital rays as possible, the reconstructed images from all primes between $p-x_{m} y_{m}$ and $p+x_{m} y_{m}$ would be averaged.

This process should be applied individually for each projection $x_{m}: y_{m}$. To avoid cycling through all $p$ projections, we choose $p^{\prime}=p+x_{M} y_{M}$ where $x_{M}: y_{M}$ is the largest product for the projections reconstructing at size $p$. This ensures the largest gap lengths are pooled sufficiently (but will also "over" average for the smaller gap lengths). Figure 4a shows a 601 by 601 image, reconstructed using the prime-based DRT, from an x-ray transmission sinogram of 511 entries at 180 uniformly spaced angles. For $p=601, x_{m}: y_{m}$ ranges from 1:24 to 17:18 so that $x_{M} y_{M}=306$. Figure 40 shows the corresponding reconstructed image result for the average of 10 images reconstructed using 10 prime array sizes from 601 to 653 . Figure 4 s shows the average of 40 reconstructed images using the 40 prime array sizes from 601 to 863 . Figure 4 d shows the same image reconstructed once but for a large array size $(p=4091)$. The projections were subsampled by 8:1 to produce a 512 by 512 final result. Figure $4 \mathrm{~d}$ is very similar to the result obtained using standard back-projection methods [7].

The reconstruction artefacts evident in Figure 4a arise from mismatching digital and analog projection rays and show the effect of the digital ray gaps. These artefacts are reduced in Figures $4 \mathrm{~b}$ and $4 \mathrm{c}$ as the pooling of digital projections produces on average a better interpolation result for each projection. The artefacts produced at each different prime image size are effectively random and cancel in the summed result.

The quality of the images in Figures $4 \mathrm{~b}-\mathrm{c}$ is still well below that obtained by more conventional reconstruction, such as Figure 4 d. The errors arise not only 
(a)

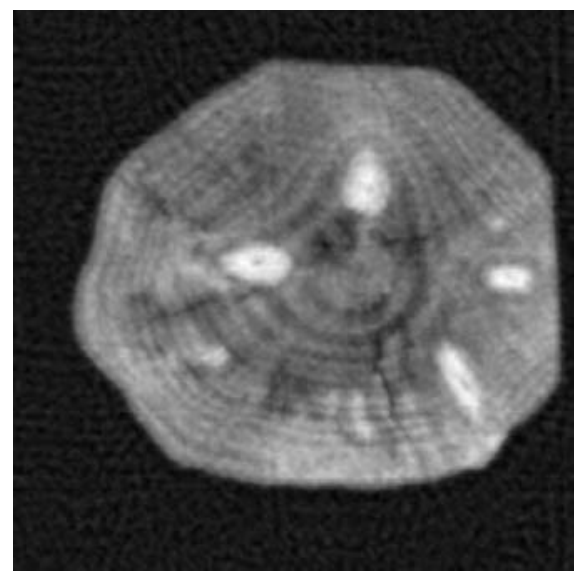

(c)

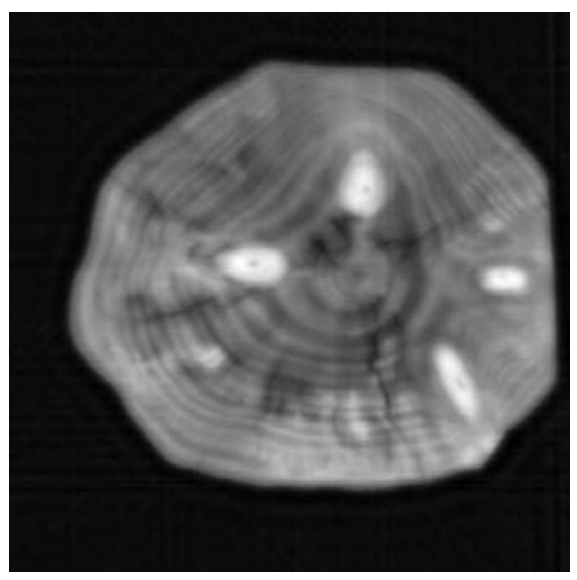

(b)

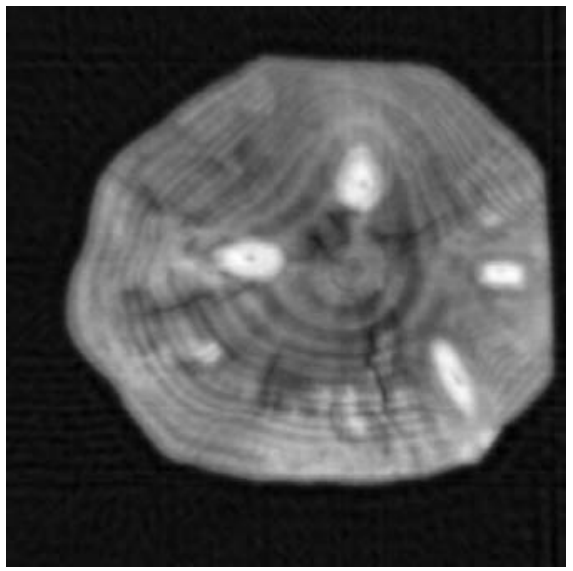

(d)

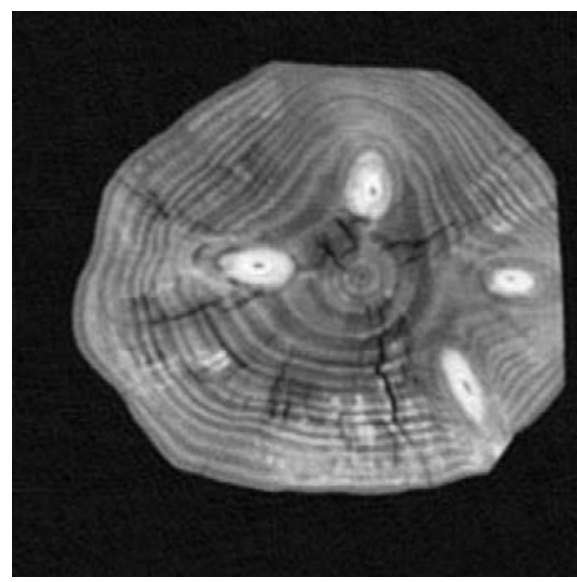

Fig. 4. a) Prime DRT image reconstructed for $p=601$, from 511 x-ray projections at each of 180 equally spaced angles. b) Average of 10 constant scale images reconstructed using the pooled DRT projection method, for primes from 601 to 653. c) Average of 40 constant scale images reconstructed using the pooled DRT projection method, for primes from 601 to 863 . d) DRT reconstruction for a single large final image format $(\mathrm{p}=4091)$, sub-sampled by $1: 8$ to produce a 521 by 512 image.

because the digital ray gaps are only partially filled, but also from the limitations of the image scaling method used to pool the digital rays. Pooling projections in the spatial or image domain requires that the image scaling and registration be as precise as possible for each prime array size to avoid blurring image details. The padding of the sinogram data was done to the nearest integral number of bins to avoid the effects of redistributing the original x-ray projection data. The pooling of digital rays should also be optimised for each $x_{m}: y_{m}$ projection value rather than over-averaging the rays with small gaps. This produces no 
additional benefit for the small gap projections but makes them very sensitive to the scale and registration problems outlined above. There was also no efficiency gain in applying the pooled image approach, as the combined time to scale and compute multiple reconstructed images, such as Figure 4r, was greater than the time required to produce a single, higher quality result from a large prime reconstructed image (such as that in Figure 4]d). See [7] for relative reconstruction times as a function of image size.

\section{Conclusions}

We have shown that digital rays from digital projections that are sampled over different prime array sizes can be pooled to better approximate integral projection rays. A distinct sampling pattern for the digital rays at each digital projection angle was associated with the uniqueness of each set of array wrap factors, $\left\{\alpha_{i}\right\}$. The limit on the range of array sizes that can be usefully pooled was established and estimates were given for the degree of gap filling by the staggered ray samples.

The pooling of digital rays through the indirect method of spatial averaging of scaled images gave some improvement in image reconstruction quality for the prime-based DRT method. However this was only enough to make this approach interesting, rather than providing a practical alternative to the "large image" DRT approximation to reduce the ray gap problem. Solving the inverse problem of direct distribution of the content of an integral projection ray amongst the component digital rays in $k$-space for any prime $p$, is considered further in 8 . Nevertheless, the relative improvement in image quality seen here offers a proof of principal that the approach of pooling digital rays does work.

\section{Acknowledgments}

IS and AK acknowledge support for this work from the Centre for X-Ray Physics and Imaging within the School of Physics and Material Engineering at Monash University. AK is a PhD student supported by an Australian Postgraduate Award provided by the Australian Government.

\section{References}

1. Beylkin, G., Discrete Radon Transform, IEEE Trans. on Acoustics, Speech and Signal Processing, vol. ASSP-35, no. 2, pp. 162-172, 1987.

2. Svalbe, I., Image Operations in Discrete Radon Space, DICTA 2002, Melbourne, Australia, Jan. 21-22, 2002, pp. 285-290.

3. Matus, F., and Flusser, J., Image Representation via a Finite Radon Transform, IEEE Transactions on Pattern Analysis and Machine Intelligence, vol. 15, no. 10, pp. 996-1106, 1993.

4. Svalbe, I., Digital Projections in Prime and Composite Arrays, IWCIA, Philadelphia, August, 2001, also see Electronic Notes in Theoretical Computer Science, www.elsevier.nl/locate/entcs/volume46.free. 
5. Svalbe, I., Sampling Properties of the Discrete Radon Transform, accepted for publication, Discrete Applied Mathematics, 2003.

6. Salzberg, P., and Figueroa, R., Chapter 19, "Tomography on the 3D-Torus and Crystals", in Discrete Tomography: Foundations, Algorithms and Applications, Eds. G. T. Herman and A. Kuba, Birkhauser, Boston, 1999.

7. Svalbe, I. and van der Spek, D., Reconstruction of Tomographic Images Using Analog Projections and the Digital Radon Transform, Linear Algebra and its Applications, 339 (2001) 125-145.

8. Kingston, A., $k$-space Representation of the Discrete Radon Transform, PhD. Thesis, School of Physics and Materials Engineering, Monash University (in preparation) 2003.

9. Svalbe I. and Kingston, A., Farey Sequences and Discrete Radon Transform Projection Angles, IWCIA'03, May 14-16, Palermo, Italy, 2003. 